\title{
Self-Alignment of Optical Fibers with Optical Quality End-Polished Silicon Rib Waveguides Using Wet Chemical Micromachining Techniques
}

\author{
Michel A. Rosa, Nam Q. Ngo, Denis Sweatman, Sima Dimitrijev, and H. Barry Harrison
}

\begin{abstract}
This paper presents a new cost-effective method for self-aligning optical fibers on silicon platforms and for achieving optical quality end-polished silicon-on-insulator (SOI) rib waveguide devices using wet chemical micromachining techniques. Through accurate alignment to the $\langle 011\rangle$ plane(s) of the (100) device layer of a SOI wafer, rib waveguide devices with selfalignment features are fabricated with the ends of each waveguide wet etched and concurrently polished providing an optical quality facet or fiber-to-waveguide interface. Eliminating the need to saw cut and then mechanically polish the ends of fabricated devices, the overall fabrication process is simplified whilst also providing an integrated optic fiber alignment capability at the ends of the fabricated waveguide devices with an alignment accuracy limited by fiber size tolerance. Experimental measurements were carried out to verify the optical quality of the waveguide facets formed using this new technique which proved excess facet losses of practically unmeasurable quantities.
\end{abstract}

Index Terms - Integrated optics, micromachining, optical planar waveguides, rib waveguides, silicon-on-insulator technology.

\section{INTRODUCTION}

$\mathbf{T}$ THE MARKET demand for cost-effective transport of large amounts of information has accelerated the development of economical and reliable optoelectronic circuits for flexible processing and distribution of high-speed signals throughout computer and communication networks. This has created a need for the well-developed low-cost microelectronics and silicon-on-insulator (SOI) technologies to be applied to the mass production of monolithically integrated optoelectronic circuits on silicon substrates to provide greater functionality for the next generation of optical networks.

Integrated optical circuits using single-mode SOI rib waveguides have recently been demonstrated as directional couplers, star-couplers, and de-multiplexers [1]-[4]. However, the waveguide ends of these circuits may not be of the highest quality because they had been saw cut and mechanically polished to achieve vertical facets, resulting in low coupling efficiency when coupled with optical fibers or other active or passive integrated optic devices. These conventional techniques are not cost-effective and not acceptable in a mass production environment. Currently, optical fiber alignment can be achieved on (100) Si wafers using V-grooves etched into the wafer's surface by either potassium hydroxide $(\mathrm{KOH})$ or a mixture of ethylene diamine and pyrocatechol (EDP), both of which

Manuscript received November 12, 1998; revised April 12, 1999.

The authors are with XEROX, Palo Alto Research Center, Palo Alto, CA 94304 USA.

Publisher Item Identifier S 1077-260X(99)07914-9. are anisotropic wet chemical etchants [5], [6]. The $\langle 111\rangle$ planes of bulk silicon act as etch stops when these and similar anisotropic etchants are used, creating $\mathrm{V}$-shaped grooves with a sidewall angle of $54.74^{\circ}$ to the wafer surface. Similar alignment dependent processing techniques are often applied to the fabrication of active and passive rib/ridge waveguide devices in order to achieve smooth sidewalls and hence minimize optical power losses [7], [8].

The $54.74^{\circ}$ sidewall angle present along the length of a single-mode waveguide structure, although not greatly affecting its modal distribution, is also present at its ends. This results in significant power losses when coupled to a cleaved fiber positioned in a V-groove structure. Attempts to overcome this have resulted in either a saw cut being made across the ends of the waveguide structure flattening the interface region [8], [9], and this can possibly introduce losses via a reduction in optical quality at the waveguide ends. Or as depicted in Fig. 1(a), the passive V-groove fiber alignment structure being kept separate from the optical device and aligned as a final step once the ends of the optical device have themselves been cut and mechanically polished to an optical quality [10]. A commonly used alternative to these two techniques is the formation of rib waveguide and associated waveguide facets via plasma etching techniques [11], [12]. Although in some cases allowing the integration of dry etched rib waveguide and wet etched V-groove on the same substrate, this process of waveguide formation still incurs additional power loss due to the inherent roughness in the dry etch process and more dominantly mask roughness that can be transferred through lithography to sidewall and facet areas of the waveguide [13].

This paper reports a unique method for fabricating via micromachining techniques and careful alignment to the $\langle 011\rangle$ plane(s) of a (100) bonded and etched back silicon-on-insulator (BESOI) wafer, an interface that can be easily adapted to any rib waveguide structure. The proposed method provides a fiber-to-waveguide self-alignment capability with an accuracy limited by fiber size tolerance and also an optical quality chemical etching and simultaneous polishing of the associated waveguide end/facet. A series of passive rib waveguide structures similar to that shown schematically in Fig. 1(b) were constructed and loss measurements were carried out to demonstrate the effectiveness and achievable optical quality of this new interface fabrication technique and to illustrate the enhanced simplicity in alignment it provides when compared to similar single-mode rib waveguide devices fabricated using the conventional saw-cut and mechanical polishing technique. 


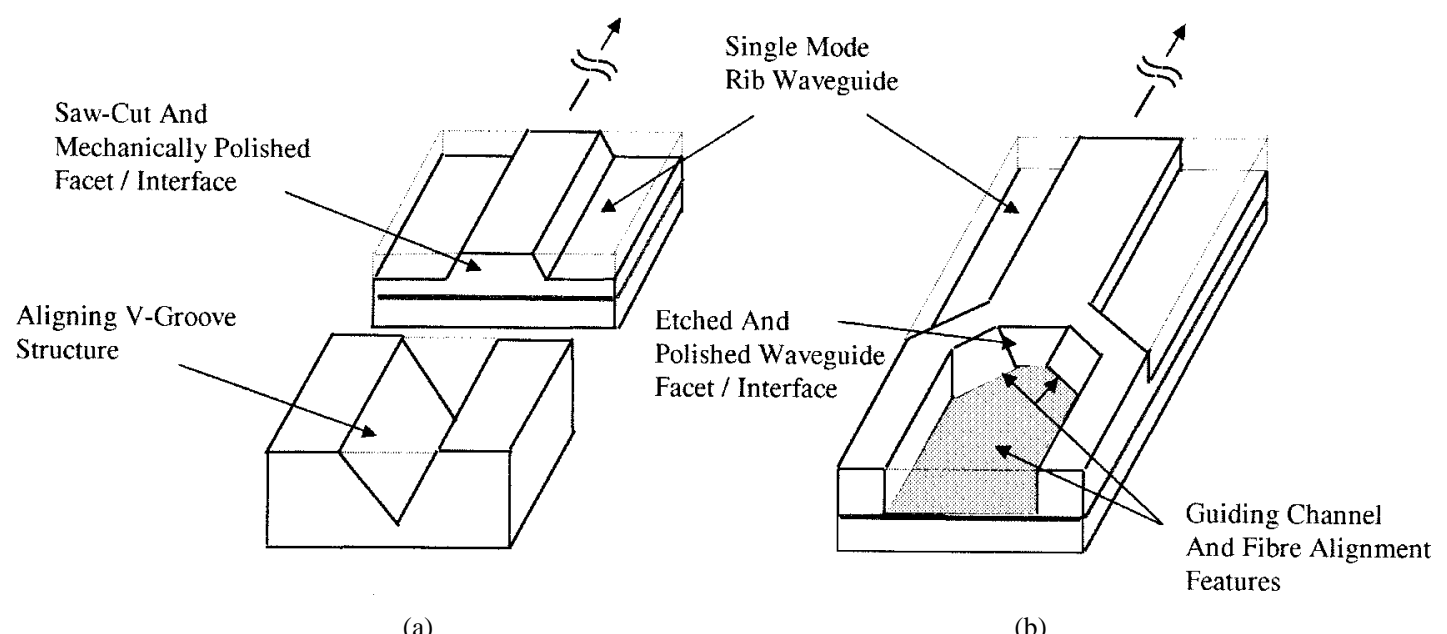

(a)

(b)

Fig. 1. (a) Standard processing techniques call for segregation of the fabricated rib waveguide device and the alignment structure whilst also having to saw cut and mechanically polish each waveguide facet in order to achieve an optically smooth surface. (b) Fabrication of both fiber alignment features and optical quality waveguide facet polishing are completed concurrently and integrated on the same substrate. The combinational advantages of this processing technique greatly reduce the power loss through misalignment errors and surface roughness on the waveguide sidewalls or end facets.

\section{RIB WAVEGUIDE FABRICATION}

A series of single-mode SOI rib waveguide devices of different lengths with the new fiber-to-waveguide interface were fabricated on a (100) n-type BESOI wafer using a two mask lithography process in conjunction with an alignment to the $\langle 011\rangle$ plane and wet chemical anisotropic etchant processing. To ensure the single-mode nature of the SOI rib waveguides fabricated using both the proposed method and conventional processing techniques, respectively, all were designed such that their heights $H$ and widths $W$ conformed to the single-mode condition [14] (where as defined in Fig. 2, $r$ is the ratio of the thickness of the side and center regions of the rib structure)

$$
\frac{W}{H} \leq 0.3+\frac{r}{\sqrt{1-r^{2}}}, \quad r \leq 0.5
$$

The cross section of both sets of waveguide devices (as defined in Fig. 2) were designed with the dimensions $H=$ $11 \mu \mathrm{m}, W=9 \mu \mathrm{m}$, and $r=0.5$ to satisfy (1) and, more importantly, to ensure an optimum coupling efficiency to a standard single-mode fiber (SMF) with a mode field diameter of $10.5 \mu \mathrm{m}$. As an aid in confirming the single-mode nature of the rib waveguide devices, numerical simulation based on the beam propagation method (BPM) of analysis was performed to calculate the mode field distribution using the physical attributes of both sets of fabricated devices. Fig. 2 shows the simulation result for the near-field intensity mode profile at the output of the rib waveguide device which is single-moded as expected.

A BESOI wafer, with a device layer thickness of $11 \mu \mathrm{m}$ and buried oxide (BOX) layer thickness of $2 \mu \mathrm{m}$, was cleaned and prepared for lithography using standard wafer preparation techniques [15]. Initial alignment to the $\langle 011\rangle$ plane was achieved using alignment marks at an angle of $45^{\circ}$ to the $\langle 100\rangle$ plane. A solution consisting of tetramethyl ammonium hydroxide (TMAH) 50\%, isopropyl alcohol (IPA) 30\%, and $\mathrm{H}_{2} \mathrm{O} 20 \%$ at $40{ }^{\circ} \mathrm{C}$ with an etch rate of $\approx 54.5 \mathrm{~nm} / \mathrm{min}$ was used to controllably etch along the $\langle 011\rangle$ plane(s) of the $\mathrm{Si}$

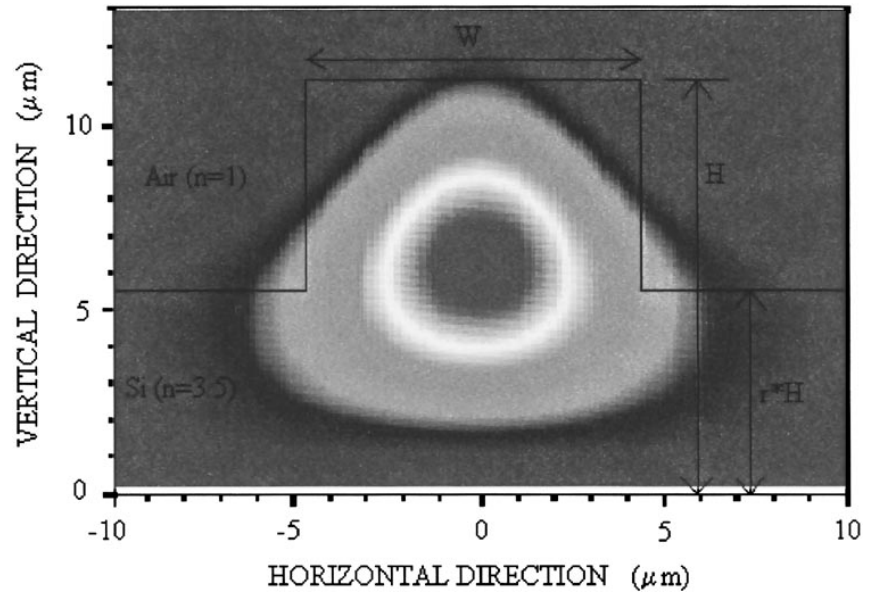

Fig. 2. Simulated view of the near-field mode distribution generated for a rib waveguide is used to verify the single-mode nature of the designed and fabricated rib waveguide devices.

device layer during device processing. Rather than using $\mathrm{KOH}$ or a solution of EDP, TMAH was chosen for its adequately high selectivity of $\mathrm{Si}$ over $\mathrm{SiO}_{2}(\approx 2000: 1)$, its relative ease of use and low toxicity [7], [16]. The SEM micrograph in Fig. 3 shows a top view of one end of the fabricated waveguide device(s), indicating which areas were fabricated during each of the two lithography and etch stages. The first lithography and timed wet etch steps defined and formed the U-groove channels for fiber alignment, along with the optical quality waveguide facets. Upon a further wafer cleaning and a subsequent re-oxidation step, a second lithography process and timed wet etch were performed. This second set of process steps resulted in the formation of the waveguide structures and their alignment with the already fabricated U-groove channels.

The SEM micrograph in Fig. 4 identifies the various materials around the end of one of the silicon rib waveguide structures. It also shows a close up view of the waveguide facet and its associated fiber alignment features. Figs. 3 and 4 demonstrate how precise alignment to the $\langle 011\rangle$ plane(s) and fine control over the wet etch chemistry used can result in a 


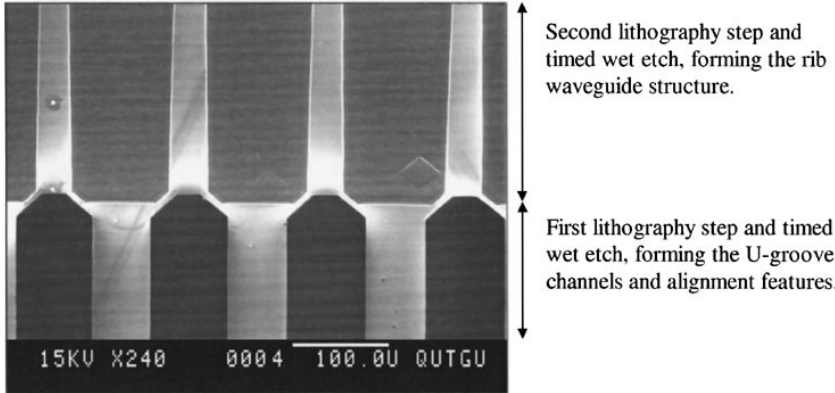

Fig. 3. Top view of the fiber-to-waveguide interface region. Added are lines indicating the parts of the device formed at each lithography step.

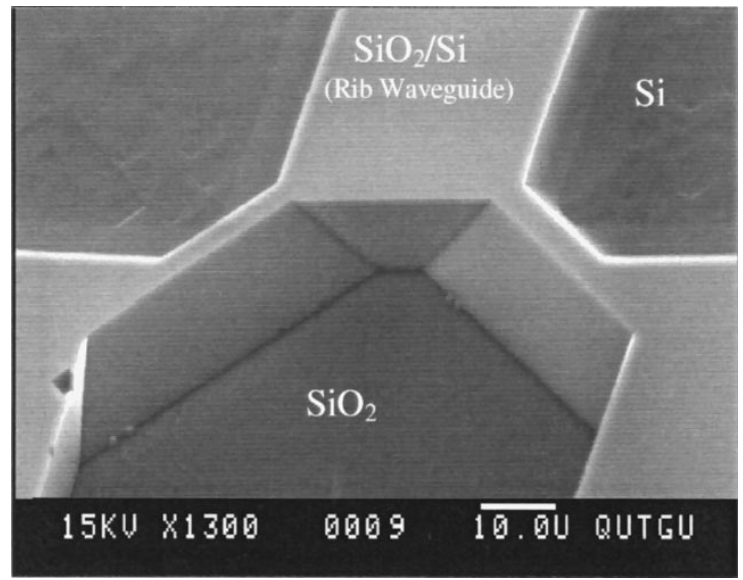

Fig. 4. Entrance to rib waveguide structure showing the polished waveguide end and the alignment features at the end of the U-groove channel.

well aligned structure with visually smooth and optically flat ends. It should however be noted that the angular dependence of the alignment step is quite large, with a misalignment $\geq \pm 1^{\circ}$ resulting in terraces forming along the etched sidewalls of the $\langle 011\rangle$ plane(s) [17]. Correct alignment is critical to ensure the waveguide facet and sidewalls are perfectly flat and maintain an optical quality smoothness. Unlike V-grooves, the U-grooves shown in Fig. 3 have vertical sidewalls and taper at their ends due to the intersection with $\langle 111\rangle$ planes. Given the precision of modern photolithography processes, the newly proposed technique enables reduction of the alignment errors to the level of fiber size tolerances. Hence, optically flat waveguide facets and a passive fiber alignment mechanism can be achieved concurrently during the fabrication of the waveguide devices themselves.

The waveguide devices shown are tailored for a SMF with a mode field diameter of $10.5 \mu \mathrm{m} \pm 0.5 \mu \mathrm{m}$. To help minimize any mode field mismatch between the coupling fibers and the rib waveguides, optical fibers of $\approx 10 \mu \mathrm{m}$ diameter were obtained through a process of timed isotropic wet chemical etching of the cladding of one end of a $125-\mu \mathrm{m}$ diameter SMF in a solution of concentrated hydroflouric acid (HF 50\%) with an experimentally determined etch rate of $\approx 1.78 \mu \mathrm{m} / \mathrm{min}$ [cf. 18]. Experimental tests have shown that retaining only a very small amount of cladding around the core of a SMF does not contribute to any significant power loss. Due to the

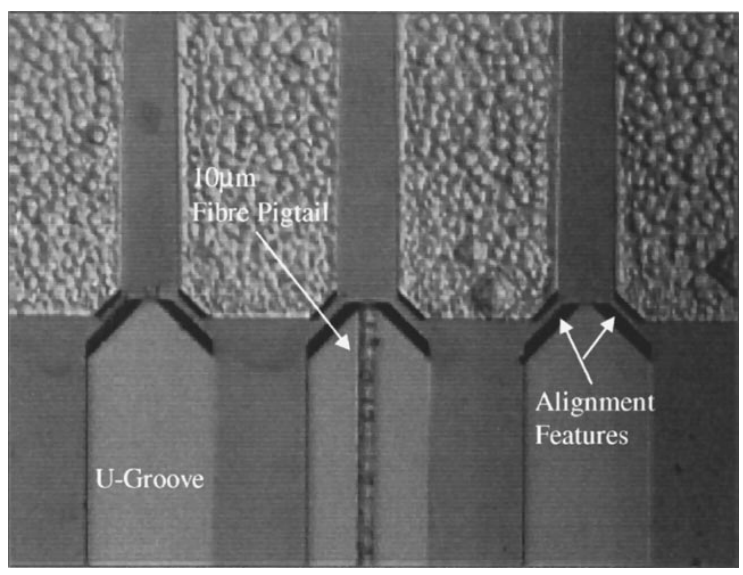

Fig. 5. A $10-\mu \mathrm{m}$ fiber is aligned to and butted up against the end of a rib waveguide structure using the U-groove and self-alignment features provided. Particulates seen along the fiber are due to the cleanliness of the atmosphere in the room at the time this optical micrograph was taken.

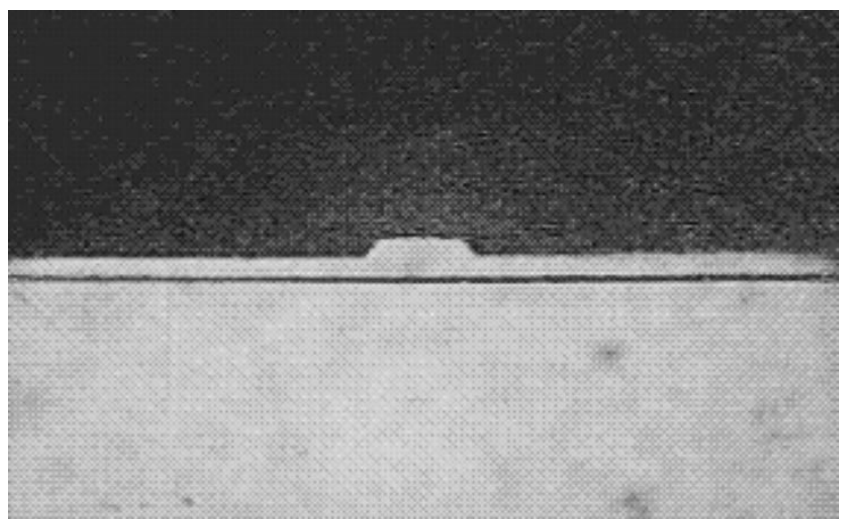

Fig. 6. Conventionally processed waveguide facet showing its smooth mechanically polished surface, the sloping $54.74^{\circ}$ sidewalls and the underlying $2-\mu \mathrm{m}$ buried oxide layer.

unavailability of (110) oriented SOI wafers of the required dimensions, thinned $10-\mu \mathrm{m}$ fibers were used so as to facilitate experimental verification of the newly proposed concepts using (100) oriented BESOI wafers. Fig. 5 shows an optical micrograph of a thinned $(\approx 10 \mu \mathrm{m})$ fiber in place at the end of one of the waveguide structures described in this section.

Device fabrication using (110) oriented BESOI wafers would result in the fabrication of an optical quality waveguide end/facet and U-groove alignment feature of dimensions such that the thinning of the $125-\mu \mathrm{m}$ cladding is no longer required. Beginning the process with a (110) oriented SOI wafer, the first mask layer can be designed to match the thickness of the fiber which is to be coupled to the waveguide. In the first lithography process step the U-groove pattern is to be aligned to the $\langle 110\rangle$ plane (similar alignment is required for subsequent waveguide patterning) so unlike fabrication using a (100) oriented substrate, the vertical etch rate becomes dominant over the lateral etch rate which in this case is impeded due to the $\langle 111\rangle$ crystallography along the walls of the etched U-groove. Once the anisotropic wet etch step commences, etching continues vertically until the buried oxide layer is reached where an additional timed wet etch 


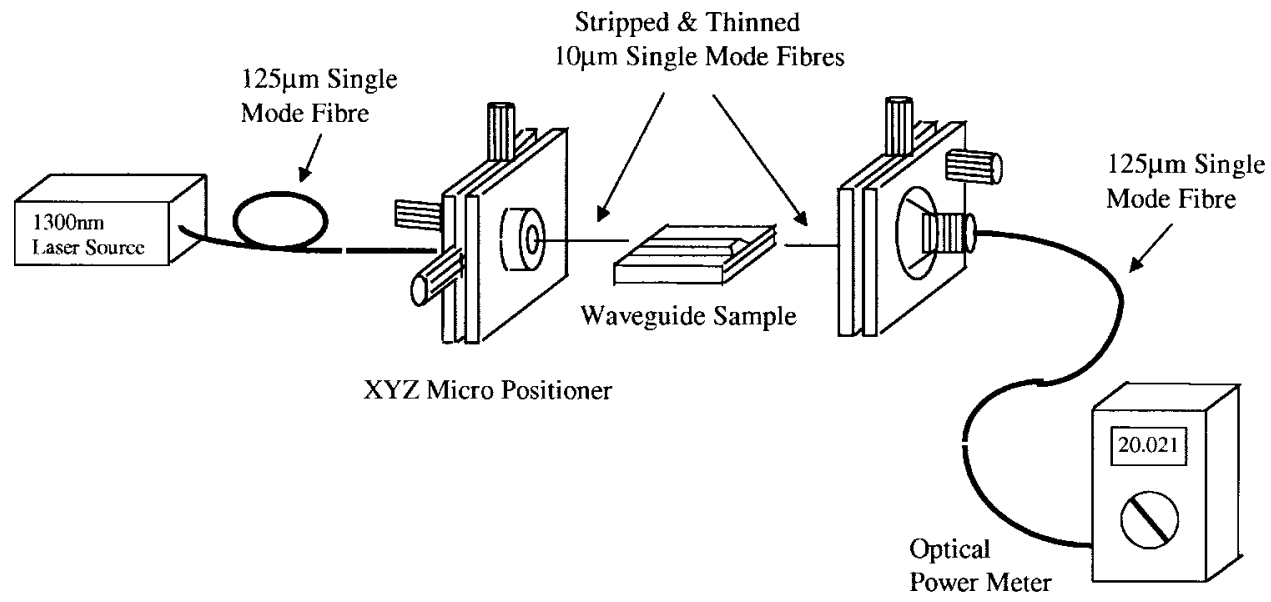

Fig. 7. Experimental setup used to test the two sets of single-mode rib waveguides fabricated using both the conventional waveguide manufacturing technique and the newly presented micromachining based technique.

in buffered HF etchant is required to remove the underlying $\mathrm{SiO}_{2}$, before the anisotropic wet etching of the U-groove recommences (etching is now at the depth of the handle wafer) and continues until the desired depth is reached. In this case, the desired depth of the U-groove channel is determined by the diameter of the fiber used and ultimately by the duration of the wet etch process. As a result of these material and process changes, the angled $\langle 111\rangle$ planes (as shown in both Fig. 3 and 4) are now removed resulting in the perpendicular termination of the U-groove structure (i.e., sidewalls) with the waveguide facet. As in previous experimental discussions, this is a timed wet etch process and can be designed to give greater fiber-to-waveguide alignment, while at the same time maintain the functionality and facet quality demonstrated in this paper. It should also be noted that changes to the fabrication of the U-groove structure in the first lithograhy and wet etch processing steps in no way alters subsequent process steps in which the waveguide itself is defined and etched. This example of the self-alignment of a fiber to a waveguide structure demonstrates the potential advantage and applicability of this method to self-align active and passive waveguide devices on the same silicon platform (i.e., monolithic integration). An example of this may be in the alignment or coupling of active and passive devices used together with flip-chip bonding techniques where laser and photodiode devices are fabricated on a multilayered substrate and later coupled to the active/passive optic waveguide circuitry which resides on a separate SOI substrate.

In order to compare this newly proposed fabrication method with existing techniques, a series of single-mode rib waveguides of varying lengths were fabricated on SOI substrates using conventional processing techniques including waveguide alignment along the $\langle 001\rangle$ plane and subsequent sawing and mechanical polishing of the waveguide ends [8], [19]. Fig. 6 shows an optical micrograph of one of the conventionally processed waveguide facets that has been saw cut and mechanically polished using $9-\mu \mathrm{m}, 1-\mu \mathrm{m}$, and finally $0.3-\mu \mathrm{m}$ grade polymer sheets coated with aluminum oxide. The loss measurements for these and the previously introduced SOI rib waveguide devices fabricated using the proposed method are discussed in the following sections.

\section{EXPERIMENTAL RESULTS AND DISCUSSION}

\section{A. Experimental Setup}

The power loss measurements were taken using an experimental setup, as shown in Fig. 7. A 1300-nm laser diode with a fiber pigtail was used to launch the light into a length of SMF having a mode field diameter of $10.5 \mu \mathrm{m} \pm 0.5 \mu \mathrm{m}$ and a core diameter of $8.3 \mu \mathrm{m}$. Two $x y z$ micro-positioners were used to manipulate the thinned $(\approx 10 \mu \mathrm{m}$ diameter $)$ end sections of both the launch and receive fibers. With the sample position fixed between both positioners, the vertical, horizontal, and angular position of the launch and receive fibers are adjusted until a maximum output power is achieved. This fine tuning is avoided almost entirely when taking power loss measurements for waveguide devices fabricated using the new fiber-to-waveguide interface. In these instances, once the send or receive fiber is placed resting in the alignment channel, the fiber can be advanced until making contact with an alignment plane located at both the left and right of the launch window (waveguide facet) where further advancement slides the fiber into the center of the optical quality waveguide facet. This precise alignment is dependent on the accuracy and control used during the fabrication of the device with which the interface is to be used. However, we have found during experimentation that it is in stark contrast to the difficulty and time incurred whilst trying to align fibers to conventionally processed waveguide ends like the one shown in Fig. 6.

\section{B. Measurement Results and Analysis}

Each set of straight rib waveguide devices varied in length from 1 to $5 \mathrm{~mm}$ and from 10 to $40 \mathrm{~mm}$ for both sets of devices fabricated using the new and conventional processing techniques, respectively. Using measurement techniques described as per the experimental setup in Fig. 7, the measured waveguide losses obtained for both sets of devices are shown in Fig. 8. The slope of the line(s) passing through the points on the main graph of Fig. 8 and its inset are identical and fixed at a value of $0.13 \mathrm{~dB} / \mathrm{cm}$ (the waveguide loss) determined mathematically using the line of best fit through the measurement results shown on the main graph. By curve fitting this line 


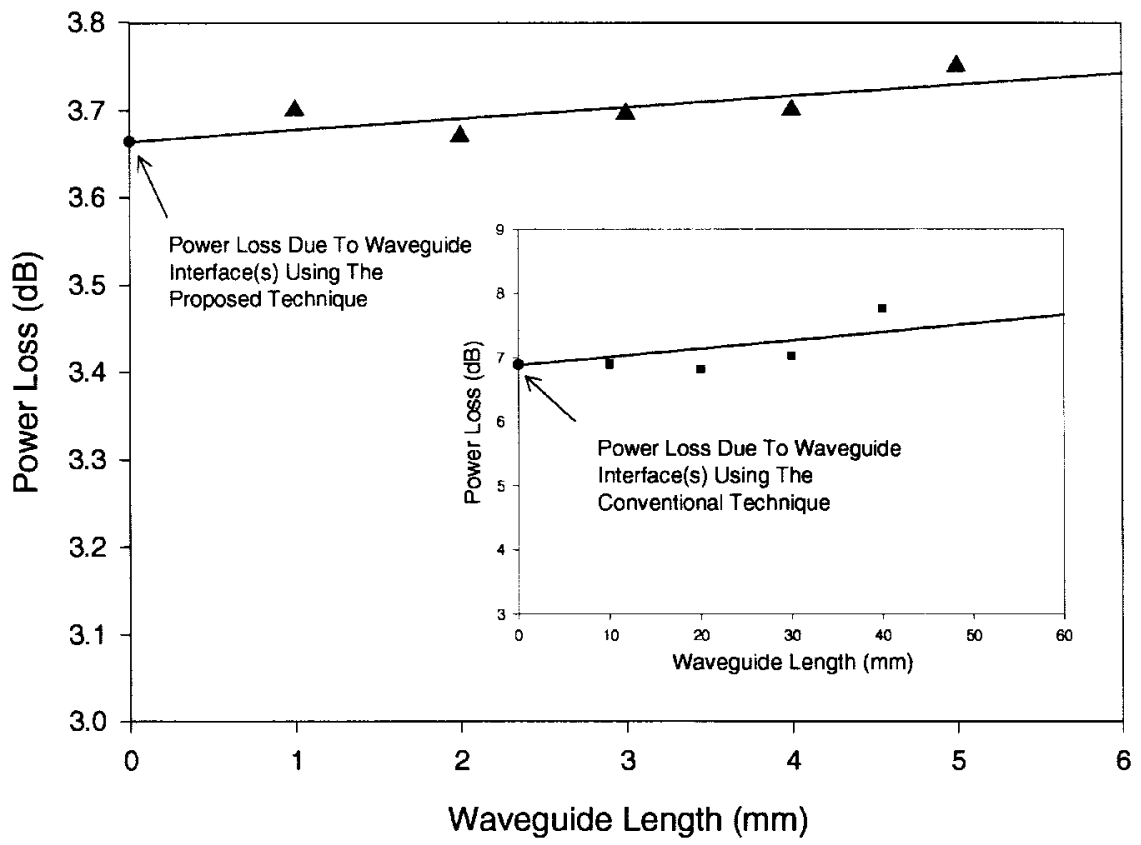

Fig. 8. Experimentally determined waveguide loss measurements showing the quality of the waveguide facet when fabricated using the new design technique. The inset shows the waveguide loss measurements using the conventional saw-cut and mechanical polishing techniques.

to the points of the inset graph in Fig. 8, the waveguide loss is assumed to be equal for all fabricated waveguide devices. Therefore, a more accurate quantitative comparison of the optical quality of the end facets for the waveguide devices fabricated using both new and old techniques can be made based solely on the loss measured due to facet roughness (assuming any measurement inaccuracy, mode field mismatch, etc., to be shared). As can be seen from the graph(s) in Fig. 8, both sets of measured results are reasonably consistent with each other and lie around this line. The line intercepts indicate power losses at the facets (power losses for "zerolength waveguide"). It can be seen from the insert-figure that our waveguide devices whose facets were fabricated using standard mechanical cut and polish techniques resulted in a measured power loss which was much higher than the theoretically calculated Fresnel Loss, which was found to be approximately $3.6 \mathrm{~dB}$. As opposed to this result, the waveguide devices fabricated using the proposed technique whose facets were wet etched resulted in a much smaller loss, so much so, that the excess facet loss becomes practically unmeasurable. The results obtained indicate a superior quality of waveguide facet having been etched and polished concurrently using the proposed fabrication method. The greater loss shown for the waveguide facets processed using the conventional saw-cut and mechanical polishing techniques, although possibly due to inconsistencies in fabrication, merely highlights the greater probability of the introduction of loss due to what appears to be a lesser quality fabrication technique. Whether the added loss is introduced via inconsistencies in mechanical polishing or fiber-to-waveguide alignment is uncertain, since both were painstakingly performed across a number of samples to verify the experimental results reported. What does remain clear, however, is the fact that the proposed fabrication technique can remove many of the inaccuracies associated with fiberto-waveguide alignment and polishing inconsistencies which lead to undue power loss at the fiber-to-waveguide interface.

\section{CONCLUSION}

This paper has introduced and discussed a unique method (which is equally applicable to the fabrication of an active device) by which a passive rib waveguide device can be fabricated with an integrated passive fiber alignment feature and optical quality waveguide facets, simply and automatically as part of the overall fabrication process. This method has been demonstrated by the fabrication and testing of a series of straight rib waveguide devices. When compared with similar devices fabricated using conventional techniques, loss measurements indicated that the optical quality of the waveguide facet produced as a result of the new wet etching process was of a higher quality and less prone to loss due to fabrication error. This new device processing technique is considered to be of great practical importance since it not only simplifies and makes more efficient a process that currently incurs an increase in production time and cost due to the added mechanical handling of the waveguide devices after their initial fabrication, but it also has been shown through experimental measurements to produce a higher optical quality waveguide facet.

\section{ACKNOWLEDGMENT}

The authors would like to thank Prof. B. Jalali and S. Yegnanarayanan of the University of California, Los Angeles, for their useful discussion. Also, thanks must go to Prof. M. Austin of RMIT for the time taken to discuss some of the issues covered in this paper. Finally, the authors would also like to thank one of the anonymous reviewers for constructive comments and suggestions. 


\section{REFERENCES}

[1] B. Jalali, P. D. Trinh, S. Yegnanarayanan, and T. Coppinger, "Guidedwave optics in silicon-on-insulator technology," Proc. Inst. Elect. Eng.-Optoelectronics, 1996, vol. 143, pp. 307-311.

[2] H. Fujita, "Application of micromachining technology to optical devices and systems," in SPIE Conf., 1996, vol. 2880, pp. 2-11.

[3] S. J. Walker, "Optics \& MEMS: An overview of current technology," in MOEMS '97, Nagoya, Japan, pp. 179-185.

[4] R. Soref, "Applications of silicon-based optoelectronics," Mater. Res. Soc. Bull., pp. 20-24, Apr. 1988.

[5] C. Strandman and Y. Backlund, "Passive alignment and holding of devices using flexible tongues formed by selective etching," in Proc. $M M E$ '96, Barcelona, Spain, pp. 113-116.

[6] M. Guendouz, N. Pedrono, J. Charrier, P. Joubert, and J. Le Rouzic, "Electrochemical micromachining of silicon platforms for optical fiber alignment," Electron. Lett., vol. 33, pp. 1695-1696, 1997.

[7] R.-S. Cheng, T.-J. Wang, and W-S. Wang, "Wet etched ridge waveguides in Y-cut lithium niobate," J. Lightwave Technol., vol. 15, pp. 1880-1887, 1997.

[8] T. Zinke and U. Fischer et al., "Comparison of optical waveguide losses in silicon-on-insulator," Electron. Lett., vol. 29, pp. 2031-2032, 1993.

[9] H. Ahlfeldt, J. Holm, S. Lindgren, L. Backlin, C. Vieider, S. Nilsson, T. Klinga, M. Nilsson, M. Svensson, L. Granlund, P. Laubert, and B. Sundstrom, "Passive alignment of laser arrays to single-mode fibers using microstructured silicon carriers," in MOEMS '97, Nagoya, Japan, pp. $155-159$.

[10] C. A. Jones and K. Cooper, "Hybrid integration onto silicon motherboards with planar silica waveguides," in Proc. Inst. Elect. Eng._Optoelectronic Devices, San Jose, CA, Feb. 13, 1997, vol. 3007, pp. $128-135$.

[11] F. Shimokawa, "New dry etching system using high-density plasma source for an optical microelectromechanical system," in MOEMS'97: Int. Conf. Optical MEMS and Their Applications, Nara, Japan, Nov. 18-21, 1997, pp. 74-79.

[12] A. V. Churnekov, "Silicon micromechanical optical waveguide for sensing and modulation," Sensors Actuators A, vol. 57 pp. 21-27, 1996.

[13] A. Harpin, "Integrated optica in silicon: Coming of age," in Proc. SPIE, Silicon-based Monolithic and Hybrid Optoelectronic Devices, San Jose, CA, Feb. 1997, vol. 3007, pp. 128-135.

[14] U. Fisher et al., " $0.1 \mathrm{~dB} / \mathrm{cm}$ waveguide losses in single-mode SOI rib waveguides," IEEE Photon. Technol. Lett., vol. 8, pp. 647-648, 1996.

[15] M. A. Rosa, S. Dimitrijev, and H. B. Harrison, "KOH wet etching techniques for the micromachining of (100) SOI wafers," in IEEE COMMAD Conf., Dec. 1996, pp. 454-457.

[16] L. M. Landsberger, S. Naseh, M. Kahrizi, and M. Paranjape, "On hillocks generated during anisotropic etching of $\mathrm{Si}$ in TMAH," IEEE J. Microelectromech. Syst., vol. 5, pp. 106-116, 1996.

[17] M. Vangbo and Y. Backlund, "Terracing of (100) Si with one mask and one etching step using misaligned V-grooves," J. Micromech. Microeng., vol. 6, pp. 39-42, 1996.

[18] H. M. Marchman, J. E. Griffith, and R. W. Filas, "Fabrication of optical fiber probes for nanometer-scale dimensional metrology," Rev. Sci. Instrum., vol. 65, pp. 2538-2541, 1994.

[19] P. D. Trinh, S. Yegnanarayanan, and B. Jalali, "Optical couplers in silicon-on-insulator technology," in Proc. Conf. Lasers and ElectroOptics, June 2-7, 1996, vol. 9, pp. 250-251.

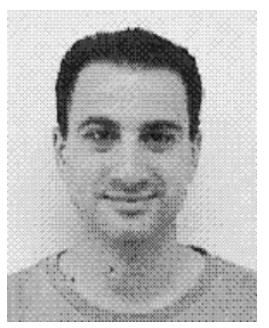

Michel A. Rosa received the B.Eng. and Ph.D degrees from the School Of Microelectronic Engineering, Griffith University, in 1996 and 1999, respectively.

Topics covered during his $\mathrm{PhD}$ research include Micro-Thermal, Micro-Mechanical and MicroOptical Devices. Currently he is a post-doctoral scientist within the Electronic Materials Lab at Xerox's Palo Alto Research Center (PARC). His current research interests include the development of Laser/MEMS integration techniques and the research of laser beam scanning technologies.

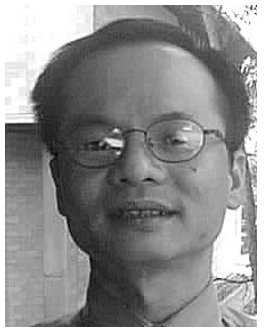

Nam Q. Ngo received the B.Eng. and Ph.D. degrees in electrical and computer systems engineering from Monash University in 1992 and 1998, respectively. In his Ph.D. study, he designed and developed a number of novel fiber-optic and integrated-optic signal processors with applications in optical computing and optical communication systems.

From 1992 to 1997, he was a microwave engineer and a research associate at Monash University. $\mathrm{He}$ has been a Lecturer at Griffith University since July 1997. His current research interests are integrated optics (in particular silicon optical waveguide devices and circuits including optical dispersion compensators), and optical communication systems and networks.

Dr. Ngois the recipient of two prizes for his Ph.D. dissertation: the Douglas Lampard Electrical Engineering Research Prize and Medal, Monash University, 1998; and the Electrical College Prize, the Institution of Engineers, Australia, 1999.

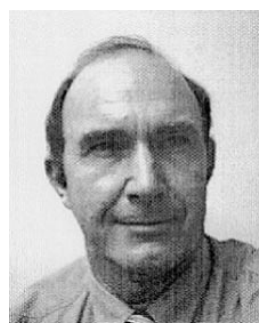

Denis Sweatman received the B.Sc. (Honors) degree in 1972 and the Ph.D. degree in 1982 in physical chemistry (spectroscopy) from the University of Queensland, Australia.

In 1982, he joined the Laser Applications Laboratory at the University of Queensland and developed holographic and infrared gas sensing systems. From 1986 to 1991, he was employed by Ionode Pty. Ltd. and developed a flow analyzer for chemical analysis. Since joining Griffith University in 1991 he commissioned the Fabrication Laboratory and researched ultrathin gate dielectrics on silicon and silicon carbide. Currently, a Lecturer in the School of Microelectronic Engineering his research interests include integrated optics, sensors, and MEMS.

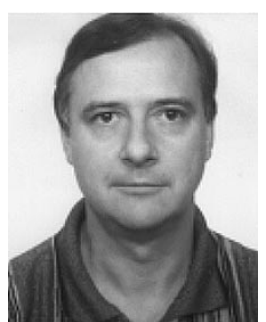

Sima Dimitrijev received B.Eng., M.Sci., and Ph.D. degrees in electronic engineering from the University of Nis in 1982, 1985, and 1989, respectively.

From 1982 to 1983, he was with the Semiconductor Factory of the Electronics Industry, Nis, where he worked on the development of CMOS technology. From 1983 to 1990 , he was with the Faculty of Electronic Engineering at the University of Nis. In 1990, he joined Griffith University where he is currently an Associate Professor at the School of Microelectronic Engineering. He is an active researcher in the areas of semiconductor technology (including micromachining and integrated optics), MOSFET applications (in particular power electronics), as well as MOSFET design, modeling, fabrication and characterization (including SiC MOSFET's). He is a member of the Editorial Board of Microelectronics Reliability (New York: Elsevier) and author of Understanding Semiconductor Devices (New York: Oxford University Press).

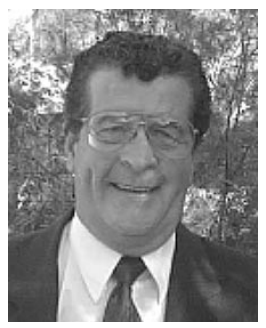

H. Barry Harrison is currently Dean of the Faculty of Engineering at Griffith University and Professor of Microelectronics. He is a Director of the Microelectronic Research Centre (formerly the Space Centre for Microelectronic Technology) and is also a major contributor to the CRC for Microtechnology. His research interests are in the area of limits to scale of integration and contact modeling. He has published in excess of 200 papers, one book, and two book chapters. 